while putrefactive decomposition could not be arrested in Nos. VII. and VIII. In one case the difference in alcoholic strength is only 0.15 per cent.

The lowest solid residue is 1.2 per eent. higher than the figure defined in the Massachusetts Statutes as the minimum for a merchantable vinegar, $i$. e. 1.5 per cent, while the average is more than double this standard.

Bostox, March 1, 1885.

\title{
TWO NEW FILTERS AND A NEW ASPIRATOK.
}

By P. Casamajor.

I propose to describe two new filters which I have lately used with advantage, and also an apparatus for starting an aspirator. 'This apparatus may be applied to one of the new filters described or to others working in a similar manner.

$$
F_{1 g} 1
$$

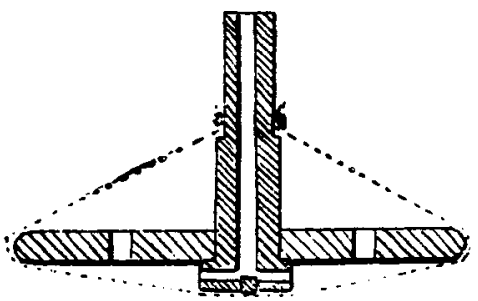

One form of filtering apparatus is shown in section in Fig. 1. The vertical portion is a tube open at the top. The horizontal portion is a circular disc, provided with a few holes to establish communication between the upper and lower surfaces of the disc. Below the disc, horizontal openings are made to allow the vertical tube to communicate with the

space below the disc. The vertical tube may have the same diameter througbout, but the walls of the tube are thicker in the lower portion than in the middle, and in this thicker than in the upper. The tube does not taper uniformly on the outside, but there is a shoulder between each portion and the one above it, the lower shoulder being designed to hold the disc in its place.

The filtering medium used with this apparatus is a circular piece of cloth, the center of which is placed under the vertical tube. The cloth is folded over the edge of the disc, so that the edges of the cloth meet above the shoulder between the middle and upper portion of the tube. These edges are fastened above this shoulder by 
means of a string or a wire. The upper portion of the vertical tube may then be connected with a rubber tube.

To use this apparstus, it may be placed in a vessel containing the liquid to be filtered. The rubber tube attached to the vertical tube may pass over the edge of the tank and be prolonged downwards on the outside below the position of the filter in the liquid. The syphon formed in this way may be started by suction. The liquid to be filtered passes through the cloth and outwards through the rubber tube.

Wood is the most convenient material for making this filter. It has the drawback that it floats, which may be remedied by attaching a weight to the portion shown in Fig. 1.

For certain purposes this portion may be made of metal-iron, lead, tin, etc. Instead of pure lead, an alloy of lead 90 parts, antimony 10 parts, tin 5 parts, will be found to be harder and sufficiently tough.

This filtering apparatus is applicable to manufacturing operations on a small scale rather than to laboratory work.

The second form of filter is one which $I$ have had in use for some time. I have named it the sand poultice. It is a bag full of sand, through which the liquid to be filtered has to pass. The bag holding the sand is made of two circular pieces of cloth, whose edges are sewn to a strip of cloth about $1 \frac{1}{2}$ inches wide. After this bag has been filled with sand, it has the shape of a flat circular box.

When in use, this sand poultice must lie on a perforated surface in a cylinder. The poultice is made of such a size that, when pressed down on the perforated surface, the vertical edge, formed by the strip of cloth, presses tightly against the cylindrical surface. It is to obtain this tight fit that the strip of cloth is used, and that the bag has not been made by sewing together the edges of the two circular pieces of cloth.

When this filter was first used, it was found impossible to dis. lodge a certain amount of air which inflated the upper covering of the sand poultice. This was afterwards avoided by making a slit in this portion of the cloth through which the air escaped. A oertain portion of dirt is in consequence deposited on the sand instead of on the cloth; but this has not been found practically objectionable.

Under the perforated plate supporting the sand poultice is a 
space for the collection of filtered liquid, which may very con veniently be let out by a fancet. With the sand may be mixed powdered charcoal, bone black, etc. The sand poultice may be taken out and washed as often as is desirable, and the bag may be refilled when neressary.

During the late period of turbidity in the Brooklyn water supply I have had occasion to use the sand poultice for filtering water for domestic use. I have found it advantageous to have a layer of fine gravel on top of the poultice, to arrest the greatest part of the muddy deposit and prevent it from obstructing the cloth. This gravel was taken out and washed twice a week without disturbing the rest of the filter. After being washed, the gravel was put back on top of the bag. After doing this five times, it was found necessary to take out the poultice itself to wash the cloth covering.

APPARATUS FOR STARTING A SYPHON.

'This apparatus was suggested by a very ingenious arrangement used in Dr. E. R. Squibl's acetic acid works. I have his permission for describing it here. In these works the acid is stored in very large stoneware receivers, which have no opening at the bottom, but they are provided with two tubulures on top. Through these tubulures pass glass tubes which go to the bottom of the receivers. Every tube is connected with a similar one on the receiver next to it by means of a bent connecting tube. These 3 tubes, held together by means of rubber tubes, form an inverted $\mathbf{V}$. On top of the bent tube is a tubulure over which passes a rubber tube, which is closed or opened by means of a pinch cock. Through this tubulure the inverted $N$ is filled by pouring in acid, after which the pinch cock is closed. The last tube of a train connects in a similar manner with a vertical glass tube which extends to the story below. This vertical tube ends, on its upper portion, in a $\mathrm{T}$, one branch of which connects with the tube in the last receiver of the train. The upper end of the $T$ has a rubber tube over it, which is closed with a pinch cock. When it is desired to draw acid from the train of receivers, this pinch cock is opened and acid is poured in a funnel above the pinch cock. The vertical tube is flled, the pinch cock closed and the acid, in running down, forms a syphon, by which the receivers are emptied, either wholly or in part, as may be desired. When sufficient acid has run out the pinch cock over the long vertical tube is opened, air is admitted and the flow ceases. 
The apparatus shown in Fig 2 is made by connecting a thistle

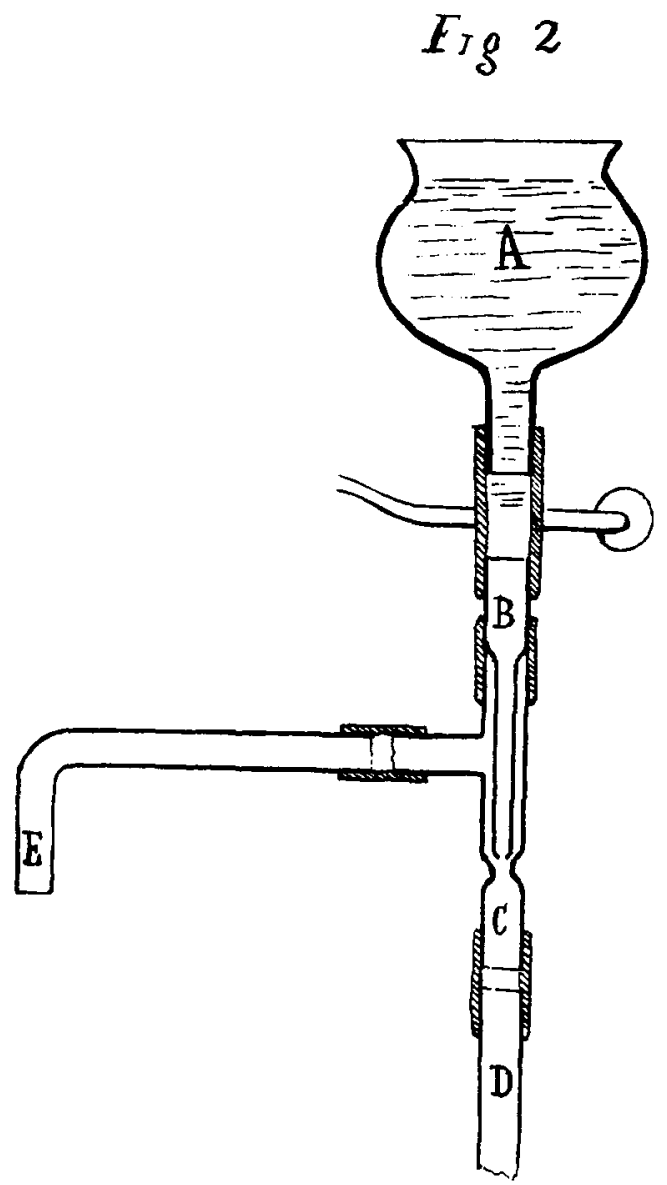
tube to a tube $B$ by means of a rubber pipe closed by. a pinch cock. The lower end of tube $B$ is placed above a reduced portion of tube $\mathrm{C}$. When water runs down from the thistle tube by opening the pinch cock, an aspiration is produced in tube $\mathrm{C}$. The horizontal branch of the $\mathrm{T}$ tube C connects with a filtering apparatus, which may be the one represented in Fig. 1. It is also applicable to Dr. Carmichael's filter, or to the filter described by me in the Journal of the Americun Chemical Society, Vol. III, p. 125, in which the vertical tube causing aspira. tion is filled by suction from a flask, but the apparatus shown in Fig. 2 is much more convenient.

After filling the thistle tube with distilled water the pinch cock is opened, and the water rushing downwards from tube B produces a vacuum in tube $\mathrm{C}$ connecting with a filter. This soon becomes filled with filtered liquid. In the apparatus in use in my laboratory 3 or 4 cubic centimeters of water are sufficient to start the action by filling the vertical tube below tube $\mathrm{C}$. 
This apparatus differs from the one used in Dr. Squibb's works, by the use of the tube $B$ and the narrowing of the tube $C$ below the end of tube $B$. This addition was found necessary because when the tube $\mathrm{B}$ is not used the opening of the pinch cock below the thistle tube causes a momentary increase of pressure in the tube $\mathrm{C}$ until the vertical tube below $\mathrm{C}$ has been filled. This disarranges the asbestos on the platinum plate in the filter described in the Journal of the American Chemical Society, Vol. III, p. 125, and the operator is put to much trouble.

\title{
ON THE ESTIMATION OF NITROGEN IN COMPOUNDS OF THE AUROMATIC SERIES BY THE KJELDAHL METHOD.
}

\author{
By J Ames H. Stebins, JR.
}

Since the publication of Kjeldahl's method for the estimation of nitrogen I have made quite a number of analyses of fertilizersin which the nitrogen was estimated according to this process, and always with good results. At first, however, I was rather doubtful of their accuracy, and therefore made a few parallel tests by combustion with soda lime. These soon showed that the method might be relied upon, except in fertilizers containing a large amount of $\mathrm{ni}$ trates. The results obtained were invariably a little bigher than those obtained by combustion, but entirely within the range of error. Having obtained such fair results with fertilizers, I was anxious to ascertain whether it might be used to advantage in the analysis of aromatic nitrogen compounds, and thus do away with the troublesome operation of estimating the nitrogen by combustion with oxide of copper.

To what extent this process may be relied upon will be seen from the following data :

$$
\begin{gathered}
\text { Paratoluidine. } \\
1: 4 \mathrm{C}_{6} \mathrm{H}_{4}\left(\mathrm{CH}_{3}\right) \mathrm{NH}_{2}
\end{gathered}
$$

0.50 grm. perfectly dry paratoluidine, was dissolved in 20 c. c. of sulphuric acid mixture (4 vols. sulphuric acid $66^{\circ}$ B., 1 vol. fuming $\mathrm{H}_{2} \mathrm{SO}_{4}$ containing in each 20 c. c. of mixture 2 grms. $\mathrm{P}_{2} \mathrm{O}_{5}$ ), and heated to boiling until the whole mixture had become nearly colorless. Time required, $1 \mathrm{~h} .15 \mathrm{~m}$. The mixture was then oxidized as usual with $\mathrm{KMnO}_{4}$, neutralized with 140 c. c. of 16 per cent soda 